\title{
Activity-Dependent Regulation of Surface Glucose Transporter-3
}

\author{
Jainne M. Ferreira, ${ }^{1}$ Arthur L. Burnett, ${ }^{2}$ and Gerald A. Rameau ${ }^{1,3}$ \\ ${ }^{1}$ Department of Biochemistry, New York University School of Medicine, New York, New York 10016, ${ }^{2}$ Department of Urology, Johns Hopkins School of \\ Medicine, Baltimore, Maryland 21287, and ${ }^{3}$ Department of Biology, Morgan State University, Baltimore, Maryland 21251
}

Glucose transporter 3 (GLUT3) is the main facilitative glucose transporter in neurons. Glucose provides neurons with a critical energy source for neuronal activity. However, the mechanism by which neuronal activity controls glucose influx via GLUT3 is unknown. We investigated the influence of synaptic stimulation on GLUT3 surface expression and glucose import in primary cultured cortical and hippocampal neurons. Synaptic activity increased surface expression of GLUT3 leading to an elevation of intracellular glucose. The effect was blocked by NMDA receptor (NMDAR) and neuronal nitric oxide synthase (nNOS) inhibition. The Akt inhibitor I (Akt-I) blocked NMDAR-induced GLUT3 surface expression while a nNOS-phosphomimetic mutant (S1412D) enhanced GLUT3 expression at cell surface. These results suggest that NMDAR/Akt-dependent nNOS phosphorylation is coupled to GLUT3 trafficking. We demonstrated that activation of cGMP-dependent protein kinase (cGK) increased the surface expression of GLUT3, which was repressed by Rp-8-pCPT-cGMPS, a potent cell-permeable inhibitor of cGKs. These studies characterize the molecular basis for activity-dependent increases in surface GLUT3 after stimulation of the NMDARs. NMDAR-induced increase in surface GLUT3 represents a novel pathway for control of energy supply during neuronal activity that is critical for maintaining glucose homeostasis during neuronal transmission.

\section{Introduction}

Glucose transporter 3 (GLUT3) is the most widely expressed glucose transporter in neurons (Nagamatsu et al., 1993; Simpson and Davies, 1994). Transgenic GLUT3 knock-out mice are nonviable, showing arrested embryonic development and embryonic lethality (Ganguly et al., 2007), while GLUT3 knockdown mutants exhibit neurologic dysfunction and altered feeding and energy balance (Schmidt et al., 2008; Zhao et al., 2010).

GLUT3 contains 12 transmembrane helices comprising a regulated central hydrophilic pore and intracellular $\mathrm{N}$ - and C-terminal ends (Dwyer, 2001). Glucose transporter activity depends on the $\mathrm{K}_{\mathrm{cat}}$, the catalytic constant of an enzyme (Simpson et al., 2007), which is sevenfold higher for GLUT3 in neurons than for GLUT1 in astrocytes (Lowe and Walmsley, 1986). The halflife of GLUT3 is $15 \mathrm{~h}$ compared with $6 \mathrm{~h}$ for GLUT1 (Khayat et al., 1998). These characteristics of the GLUT3 make it well suited for the high energy demand of neurons.

The NMDA receptor (NMDAR) is critical for activitydependent plasticity. $\mathrm{Ca}^{2+}$ flux through the NMDAR pore filter domain activates neuronal nitric oxide synthase (nNOS), which produces nitric oxide (NO). One of the primary targets of NO in neuronal cells is guanylate cyclase (GC), which produces cGMP to activate cGMP-dependent protein kinase (cGK).

Received April 17, 2009; revised Nov. 3, 2010; accepted Dec. 6, 2010.

This work was supported by a National Institutes of Health Grant Supplement 3R01DK64679-03. Special thanks to Dr. Edward Ziff and Joe Hurt for a close reading of the manuscript, and Dr. Mark Mattson for helpful discussions.

Correspondence should be addressed to Gerald A. Rameau, Department of Biology, Morgan State University, Baltimore, MD 21251. E-mail: rameag01@yahoo.com.

DOI:10.1523/JNEUROSCI.1850-09.2011

Copyright $\odot 2011$ the authors $\quad 0270-6474 / 11 / 311991-09 \$ 15.00 / 0$
cGKII regulates the AMPA receptor GLUR1 subunit trafficking to the plasma membrane via phosphorylation at S845 (Serulle et al., 2007).

We hypothesized that synaptic activity may regulate the surface expression of GLUT3 by a NO/cGK-dependent mechanism. We examined the expression of surface GLUT3 after treating neurons with bicuculline plus 4-aminopyridine (4-AP). In neurons, both $\mathrm{Ca}^{2+}$ influx via the NMDAR and nNOS phosphorylation at serine 1412 stimulate nNOS activity and increase NO production (Bredt and Snyder, 1989; Garthwaite et al., 1989; Rameau et al., 2007). Since nNOS serine 1412 phosphorylation stimulates NMDAR-induced NO production (Rameau et al., 2007), the coupling of this regulatory phosphorylation to GLUT3 trafficking was also examined. Furthermore, inhibition of the cGK pathway blocks NMDAR-induced expression of surface GLUT3. Together, these results suggest a direct relationship between increased synaptic activity, elevated expression of surface GLUT3, and glucose transport.

\section{Materials and Methods}

Cell culture. Cultured embryonic day (E) 18 rat cortical and hippocampal neurons were prepared as described previously (Rameau et al., 2000). Embryos were obtained from pregnant Sprague Dawley rats. Embryonic brains were excised on E18 with a forceps and put in a tissue culture dish containing cold buffer. Under a dissecting microscope, the cerebellum and the brainstem were separated from the brain. The hippocampi were pooled separately from the cortices, and the rest were discarded. Whole cortical and hippocampal tissues were digested in PBS-HEPES-glucose dissecting solution containing $0.05 \%$ trypsin-EDTA for $10 \mathrm{~min}$ at $37^{\circ} \mathrm{C}$. The neurons were dissociated from the tissues by gentle repeated passages through a $10 \mathrm{ml}$ pipette. Tissue culture dishes $(60 \mathrm{~mm})$, previously 
coated with poly-L-lysine, received $3.0 \times 10^{6}$ cells in $5 \mathrm{ml}$ of MEM plus $10 \%$ horse serum and $0.45 \%(\mathrm{w} / \mathrm{v})$ glucose. Coverslips $(22 \times 22 \mathrm{~mm})$ installed in a 6 well dish each received $1.5-2 \times 10^{5}$ cells in $3 \mathrm{ml}$ of minimum essential Eagle's plating medium (Eagle) (MEM). All reagents, unless stated otherwise, were obtained from Invitrogen.

Neurons were incubated at $37^{\circ} \mathrm{C}, 5 \% \mathrm{CO}_{2}$ in $5 \mathrm{ml}$ of MEM for $3 \mathrm{~h}$, after which the plating medium was removed and replaced with Neurobasal medium supplemented with B27, $0.5 \%$ horse serum, and antibiotics. At $5 \mathrm{~d}$ in vitro (DIV), neurons were fed with fresh Neurobasal medium containing $5 \mu \mathrm{M}$ uridine and $5 \mu \mathrm{M} 5$-fluor-2'-deoxyuridine (FdU) to prevent the growth of non-neuronal cells. Cells were fed once every week.

Madin-Darby canine kidney (MDCK) cells were obtained from the American Type Culture Collection and were grown in MEM containing $10 \%$ fetal bovine serum (FBS), 2 mM L-glutamine, $1.5 \mathrm{~g} / \mathrm{L}$ sodium bicarbonate, $0.1 \mathrm{~mm}$ nonessential amino acids, and $1 \mathrm{~mm}$ sodium pyruvate at $37^{\circ} \mathrm{C}$ and $5 \% \mathrm{CO}_{2}$. Cells were split $1: 8$ once a week $(0.25 \%$ trypsin- 0.53 mM EDTA), and medium was changed every $2 \mathrm{~d}$.

The human embryonic kidney cell line HEK293T, which contains the SV40 large T antigen (HEK 293T), was cultured in DMEM supplemented with $10 \%$ FBS, $2 \mathrm{~mm}$ L-glutamine, and $100 \mu \mathrm{g} / \mathrm{ml}$ streptomycin at $37^{\circ} \mathrm{C}$ and $5 \% \mathrm{CO}_{2}$. Cells were split 1:10 twice a week (0.5\% trypsin- $0.53 \mathrm{~mm}$ EDTA), and medium was changed every $2 \mathrm{~d}$.

MDCK and HEK 293T cells, after the fifth passage, were plated on coverslips at a density of 50,000 cells $/ 2 \mathrm{ml}$ and allowed to reach $70-80 \%$ of confluence before they were used for immunofluorescent labeling.

Cell culture infection. Sindbis virus expressing green fluorescent protein (GFP) or myc-epitope-tagged nNOS (wild-type and S1412D) was applied to hippocampal cells cultured on coverslips or cortical cells cultured in $60 \mathrm{~mm}$ dishes. Infection was performed overnight; the next day, cells were used for biotinylation assay or immunofluorescence.

Surface biotinylation and precipitation. Cortical neuron cultures 15-21 DIV were washed twice with $2 \mathrm{ml}$ of cold PBS. A solution of freshly made Sulfo-NHS-SS-biotin (Pierce) $1.0 \mathrm{mg} / \mathrm{ml}$ dissolved in PBS was applied on the cells for $12 \mathrm{~min}$. The binding reaction was quenched with $100 \mu \mathrm{l}$ of quenching solution (Pierce). Cells were washed three times with $2 \mathrm{ml}$ of cold PBS. The cells were gently scraped and transferred into a $1.5 \mathrm{ml}$ microcentrifuge tube, and pelleted by centrifugation at $4000 \mathrm{rpm}$ for 2 $\mathrm{min}$ at room temperature. The PBS solution was removed, and the cells were lysed in $150 \mu \mathrm{l}$ of radioimmunoprecipitation assay (RIPA) buffer rocked for $1 \mathrm{~h}$ at $4^{\circ} \mathrm{C}$, then centrifuged at $14,000 \mathrm{rpm}$ for $15 \mathrm{~min}$. The supernatant was transferred to a new tube containing $100 \mu \mathrm{l}$ of immobilized NeutrAvidin gel slurry (Pierce). Cell lysates were incubated in the rocking solution for $2 \mathrm{~h}$ at $4^{\circ} \mathrm{C}$. The lysates were centrifuged at $8000 \mathrm{rpm}$ for $2 \mathrm{~min}$ to remove the supernatant. The slurry was washed twice with 1 $\mathrm{ml}$ of RIPA buffer containing $500 \mathrm{~mm} \mathrm{NaCl}$ and once with RIPA buffer containing $150 \mathrm{~mm} \mathrm{NaCl}$. This was followed by centrifugation at 4000 rpm for 2 min to remove excess buffer and resuspension of the slurry in $50 \mu$ of $2 \times$ SDS-PAGE sample buffer containing $50 \mathrm{~mm}$ DTT. After these procedures, the lysates were boiled for $10 \mathrm{~min}$ and analyzed by Western blots.

Synaptosome preparation. The nucleus accumbens from adult rats was removed and placed in ice-cold solution A $(0.32 \mathrm{~m}$ sucrose, $1 \mathrm{~mm}$ $\mathrm{NaHCO}_{3}, 1 \mathrm{~mm} \mathrm{MgCl}, 0.5 \mathrm{~mm} \mathrm{CaCl}$ ), $0.1 \mathrm{~mm}$ PMSF (Sigma) and $1 \times$ Complete Protease Inhibitors (Roche Applied Science). Tissue was Dounce homogenized in $40 \mathrm{ml}$ of solution A for each $10 \mathrm{~g}$ of wet nucleus accumbens tissue. The homogenates were diluted to $10 \% \mathrm{w} / \mathrm{v}$ with solution $\mathrm{A}$ and centrifuged at $1400 \times g$ for $10 \mathrm{~min}$. The supernatant solution was saved, and the pellet was rehomogenized in $10 \%$ solution A per $10 \mathrm{~g}$ of initial weight and subjected to a centrifugation at $710 \times g$ for $10 \mathrm{~min}$. Supernatants were pooled and subjected to a second centrifugation at $710 \times g$ for $10 \mathrm{~min}$. The supernatants were then spun at $30,000 \times g$ for 15 min to obtain a crude P2 fraction. The pellet was resuspended in solution $\mathrm{B}\left(0.32 \mathrm{M}\right.$ sucrose, $\left.1 \mathrm{~mm} \mathrm{NaHCO}_{3}\right)$ using $24 \mathrm{ml}$ of solution $\mathrm{B}$ per $10 \mathrm{~g}$ of starting material. This homogenate was layered on top of a $5.5 \mathrm{ml}$ of $1 \mathrm{M}$ sucrose and $5.5 \mathrm{ml}$ of $1.2 \mathrm{M}$ sucrose gradient, and was centrifuged at $82,500 \times g$ for $2 \mathrm{~h}$. Purified synaptosomes were collected at the 1 and $1.2 \mathrm{~m}$ sucrose interface by syringe aspiration. Synaptosomes were resuspended with 4 volumes of solution B and collected by centrifugation at $48,200 \times g$ for $30 \mathrm{~min}$. Pellet was lysed in $100 \mu \mathrm{l}$ of $2 \%$ SDS in $25 \mathrm{~mm}$ Tris.

Western blots. SDS-PAGE and Western blots were performed essentially as described previously (Rameau et al., 2007). Antibodies were used at the following dilutions: anti-GLUT3 (C terminal, 1:1000 dilution; ab41525, Abcam); anti-phosphoS1412nNOS and anti-phosphoS847nNOS (1:1000 dilution) (Rameau et al., 2007); anti- $\beta$-actin (1:1000 dilution; A-2066, Sigma); anti-c-MYC (1:1000 dilution; sc-40, Santa Cruz Biotechnology); anti-nNOS (1:1000 dilution; catalog \#610308, BD Biosciences); anti-NMDAR1 (0.5 $\mu \mathrm{g} / \mathrm{ml}$; MAB 363, Millipore Bioscience Research Reagents); anti-postsynaptic density protein 95 (PSD-95) (1: 2000 dilution; catalog \#73028, NeuroMab). Polyclonal and monoclonal antibodies were detected by PICO-ECL (Pierce). Densitometric analysis of Western blots was performed with National Institutes of Health Image software.

Immunostaining. For immunostaining, neuronal cultures were fixed and blocked as described previously (Rameau et al., 2003). The same procedure was used for HEK 293T and MDCK cells. For surface staining, incubation with anti-GLUT3 (N-terminal extracellular domain, 1:200 dilution; sc-31838, Santa Cruz Biotechnology) was performed overnight at $4^{\circ} \mathrm{C}$ without cell permeabilization. For intracellular staining, incubations with anti-c-MYC (1:250 dilution; sc-40, Santa Cruz Biotechnology); anti-microtubule-associated protein 2 (MAP2) (1:300 dilution; 05-346, Millipore); anti-synaptophysin (1:300 dilution; S 5768, Sigma); and anti-GLUT3 (C terminal, 1:250 dilution; ab-41525, Abcam) were performed after cells were permeabilized with $0.1 \%$ Triton X-100 for 5 min at room temperature. All secondary antibodies were applied for $1 \mathrm{~h}$ at room temperature and were conjugated to Alexa Fluor 488, Alexa Rhodamine Red-X (1: 2000 dilution, Invitrogen), Rhodamine Red-X, Cy5 (Cy5), or fluorescein isothiocyanate (FITC) (1:300 dilution, Jackson Immunoresearch). Membranes of MDCK and HEK 293T cells were stained with the lipophilic dye DiI C18 (1, $1^{\prime}$-dioctadecyl-3,3,3', $3^{\prime}$ tetramethylindocarbocyanine perchlorate, D-282, Invitrogen/Invitrogen). Images were acquired using an inverted Zeiss LSM 510 laser-scanning confocal microscope or a Nikon PCM 2000 confocal microscope, and analysis was performed blind to the treatment of cells using ImageJ software. Images for all experimental groups were taken using identical acquisition parameters. With images acquired for quantification, background image intensity was determined and subtracted by thresholding during image acquisition. For surface and intracellular GLUT3, the intensity of the antibody-conjugated-fluorochromes (Rhodamine Red-X, or FITC, Jackson Immunoresearch) was measured in primary dendrites within boxes of identical size using identical settings of ImageJ software or Zeiss software. To measure the ratio of surface to intracellular GLUT3, the intensities of both pools were measured from the same dendritic box. Fluorescence measurements from an average of 10-20 neurons per coverslip were averaged to obtain a population mean (presented as mean \pm SEM). Statistical significance of differences between means was determined by Student's $t$ test or ANOVA followed by Bonferroni test, using the software GraphPad Prism.

Hexose uptake. Hippocampal cultures were rinsed with Krebs-RingersHEPES (KRH) buffer supplemented with $3.3 \mathrm{~mm}$ glucose. The uptake of 2-[N-(7-nitrobenze-2-oxa-1, 3 diazol-4-yl) amino]-2 deoxy-glucose (2NBDG), a fluorescent glucose tracer was used to measure glucose transport, 2-NBDG $(600 \mu \mathrm{M})$ was added to the KRH buffer. To study the effect of NOS inhibition on glucose uptake, $\mathrm{N}^{\omega}$-nitro-L-arginine $\left({ }^{\omega} \mathrm{NA}\right)$, a broad inhibitor of NOS, was added $10 \mathrm{~min}$ before 2-NBDG uptake. The cells were washed three times with PBS to remove free 2-NBDG from the extracellular space and fixed with $4 \%$ paraformaldehyde. Accumulation of intracellular 2-NBDG, excited at $488 \mathrm{~nm}$, was imaged using an inverted Zeiss laser-scanning confocal microscope at $20 \times$ magnification. The area defined by labeling with wheat germ agglutinin (WGA) dye defined regions of interest, and nonspecific labeling was eliminated by size exclusion.

Statistical analysis. Data are presented as means \pm SEM. Statistical analysis was performed using the unpaired Student's $t$ test and ANOVA with post hoc Bonferroni/Dunn test, using Stat View (Abacus Concepts). 

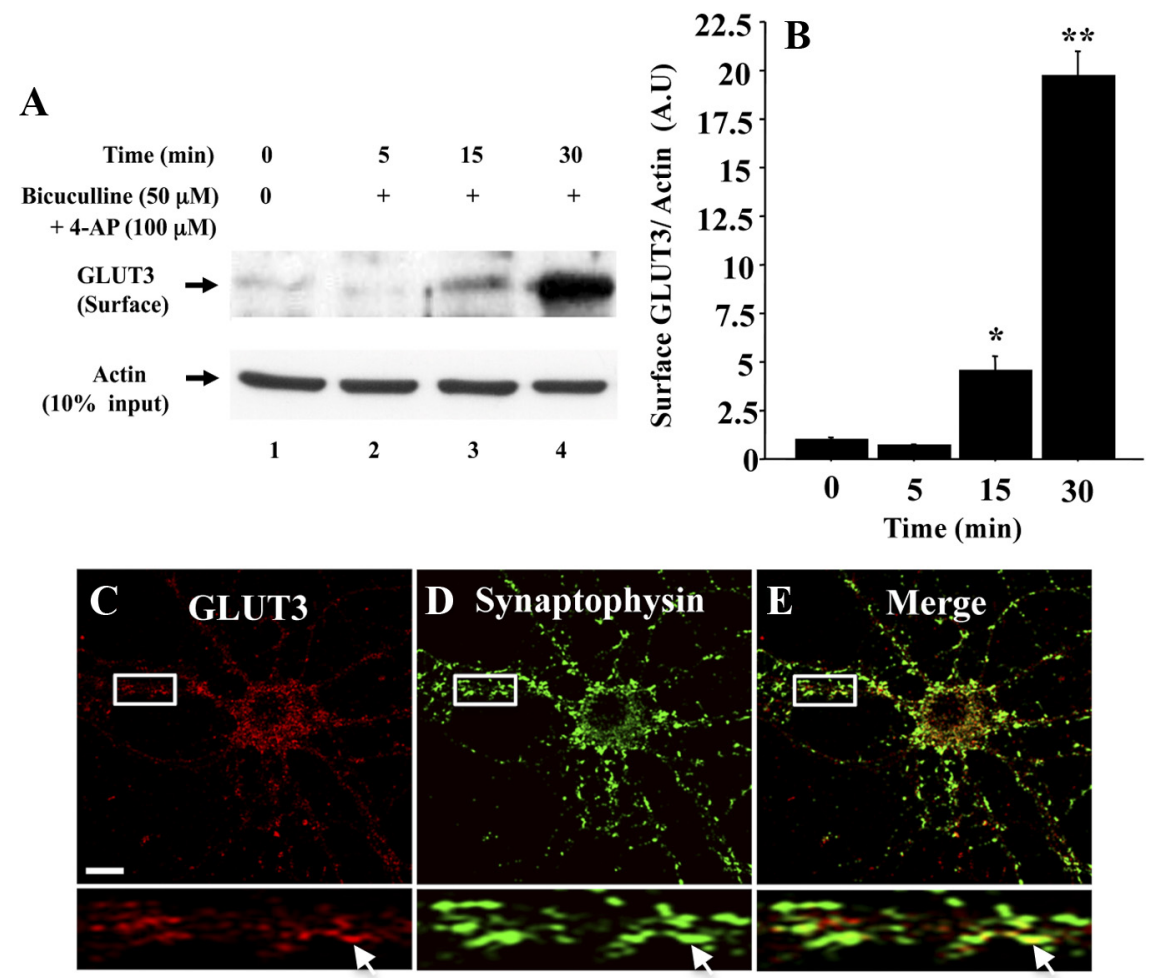

$\mathbf{F}$

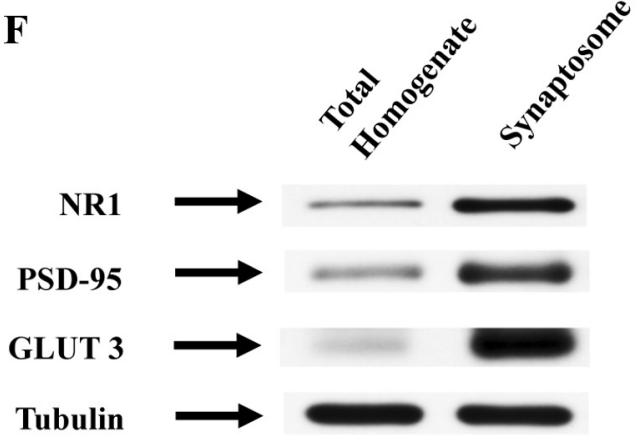

Figure 1. Increased synaptic activity augments GLUT3 expression at cell surface. $\boldsymbol{A}$, Time course of increased GLUT3. Western blots for actin confirm equal protein loading in each lane. $B$, Quantification of GLUT3 was performed by determining the ratio of GLUT3 to actin. C-E, GLUT3 expression was examined in hippocampal neurons immunostained with antibodies against total GLUT3 (red label; rhodamine) (C), synaptophysin (green label; fluorescein) (D), and merged images showing the close proximity of synaptophysin and GLUT3 punctae (E). Scale bar, $20 \mu \mathrm{m}$. F, GLUT3 localization in synaptosomal preparations from the nucleus accumbens. NR1, PSD-95, and GLUT3 Western blot of total tissue homogenates and synaptosomes. A.U., Arbitrary units. ${ }^{*} p<$ $0.001 ;{ }^{* *} p<0.0001$.

\section{Results}

Synaptic activity increases the surface expression of GLUT3

Synaptic transmission depends, in part, on glucose to satisfy the high energy demand of neuronal cells. To assess the mechanism by which synaptic activity regulates surface GLUT3 expression, we treated cortical neuronal cultures with $50 \mu \mathrm{m}$ bicuculline and 100 $\mu \mathrm{M} 4$-AP. This treatment caused bursts of action potentials (Hardingham and Bading, 2002). Surface proteins were then isolated by biotin-streptavidin pulldown and analyzed by Western blot. Treatment with bicuculline plus 4-AP induced a gradual increase in surface GLUT3, 5-fold at $15 \mathrm{~min}$ and 20-fold at $30 \mathrm{~min}\left(n=3\right.$; ${ }^{\star} p \leq$ $\left.0.001,{ }^{*} p \leq 0.0001\right)$ relative to the unstimulated control, indicating that synaptic activity increased surface GLUT3 (Fig. $1 A, B$ ).

Although synaptic activity appeared to regulate GLUT3 surface expression, the presence of GLUT3 at functional synapses had not been verified. Immunostaining of hippocampal neurons with antibodies against the C-terminal domain of GLUT3 (red label; rhodamine) and synaptophysin (green label; fluorescein) showed GLUT3 staining in dendrites and in the cell body (Fig. 1C); synaptophysin staining was noted in presynaptic nerve terminals (Fig. $1 D)$. Merged images show the juxtaposition of synaptophysin and GLUT3 punctae (Fig. $1 E$ ), which suggests a synaptic location of GLUT3. To confirm the localization, we dissected the nucleus accumbens from adult rat brains and prepared total and synaptosomal fraction homogenates for Western blotting. GLUT3 was highly expressed in synaptosomes; Western blots for PSD95 (a postsynaptic density marker) and NR1 (NMDAR subunit1) confirmed the successful isolation of synaptosomes (Fig. 1F).

Distribution of surface and cytoplasmic GLUT3 in cultured hippocampal neurons

We determined the ratio of surface to intracellular GLUT3 in hippocampal neuronal cultures using antibodies against the first extracellular loop (N-GLUT3) and the C-terminal domain of GLUT3 (CGLUT3). First, we characterized the antibodies for immunostaining using HEK293T and MDCK cells. As expected in MDCK cells, which do not express GLUT3, the N-GLUT3 and C-GLUT3 antibodies showed no labeling for GLUT3 (Fig. 2E). In contrast, HEK293T cells, which express GLUT3, showed strong labeling with both $N$-GLUT3 and C-GLUT3 antibodies. The expression pattern of surface GLUT3 was consistent with the restricted location of cell surface transporters, while labeling for total GLUT3 showed a broader and more diffuse distribution (Fig. 2D), consistent with the expected staining patterns. As a further control, labeling using the antibody directed against the intracellular C-terminal domain of GLUT3 was absent in cells that were not detergent permeabilized (Fig. 2C).

In day 21 cultured hippocampal neurons, the levels of GLUT3 were $0.714 \pm 0.0358(n=60)$ at the dendritic surface relative to $1.13 \pm 0.0768(n=60)$ intracellular levels in nonstimulated cells. This implies a substantial pool of GLUT3 available for translocation to the cell surface. Then, we determined the ratio of surface to intracellular GLUT3 in dendrites of individual hippocampal neurons after stimulation with bicuculline and 4-AP for $15 \mathrm{~min}$. After stimulation, surface GLUT3 increased from $0.505 \pm 0.031$ to $0.916 \pm 0.094(n=60, p \leq 0.0001)$. Representative images of stimulated and vehicle-treated cells are shown in Figure 2, $A$ and $B$.

NMDAR signaling regulates the surface expression of GLUT3 via NO

To examine whether NMDAR influences GLUT3 trafficking, surface-expressed proteins were isolated from cultured cortical 
neurons by the biotin-streptavidin pulldown assays. After treatment with bicuculline plus 4-AP for $15 \mathrm{~min}$, surface GLUT3 increased approximately fivefold $\left(n=3 ;{ }^{\star} p=0.005\right)$. This was blocked by $30 \mu \mathrm{M}$ MK801 [(5S,10R)-(+)-5-methyl-10, 11-dihydro-5H-dibenzo $[a, d]$ cyclohepten5,10-imine maleate], an NMDAR openchannel blocker, and by $100 \mu \mathrm{M}{ }^{\omega} \mathrm{NA}$, a nonselective broad nNOS inhibitor (Fig. $3 A, B)$. Examination of surface GLUT3 by quantitative confocal microscopy in cultured hippocampal neurons confirmed the biochemical observations. After stimulation, surface GLUT3 increased fourfold relative to vehicle-treated control (Fig. 3C, E, F). This increase was blocked by the NMDAR antagonist (2R)-amino-5phosphonopentanoate (AVP) $100 \mu \mathrm{M}$ (Fig. $3 D, F)$. Together, these results show that increased surface GLUT3 after stimulation depends on NMDAR activation and NMDAR/NO signaling.

Nitric oxide increases surface GLUT3 To assess the role of NO in the expression of surface GLUT3, cultured cortical neurons were incubated with the NO donor ( \pm$)-(E)$-ethyl-2-[(E)-hydroxyimino]5-nitro-3-hexeneamide (NOR-3). After treatment with $50 \mu \mathrm{M}$ NOR-3, surface GLUT3 increased 15-fold at $15 \mathrm{~min}$, remaining at that level for $30 \mathrm{~min}$, relative to control (Fig. 4A,B). This observation was then confirmed by quantitative confocal microscopy in cultured hippocampal neurons. We found that surface GLUT3 increased eightfold ( $n=50, p \leq 0.001$ ) (Fig. 4C,F) relative to vehicle controls or a 5 min incubation with NOR-3 (Fig. $4 D_{-}$ $F)$. These results suggest that NO is sufficient to induce surface expression of GLUT3.

\section{Synaptic activity regulates NMDAR-induced sequential phosphorylation of nNOS}

Previously, we showed that neuronal stimulation with $30 \mu \mathrm{M}$ glutamate leads to biphasic phosphorylation of nNOS at S1412 and S847 (Rameau et al., 2007). Since stimulation with bicuculline and 4-AP increased GLUT3 surface levels via NO, we investigated the differential phosphorylation status of nNOS in cortical neurons. After stimulation with bicuculline plus 4-AP and Western blot analysis with antibodies against nNOS$\mathrm{S} 1412-\mathrm{PO}_{4}$ and $\mathrm{S} 847-\mathrm{PO}_{4}$ (Rameau et al., 2007), neurons showed a biphasic increase in phosphorylation of nNOS at S1412 and S847. The phosphorylation of S1412 increased 5 -fold over basal levels at $15 \min (n=3, p \leq 0.003)$, followed by a decline and then a second increase at 45-60 min that reached a maximum of $\sim 20$-fold over the basal levels $(n=3$, $p \leq 0.0001$ ) (Fig. 5A,C). Incubation with MK801 (30 $\mu \mathrm{M}, 15$ min) blocked the phosphorylation at $\mathrm{S} 1412$ (Fig. 5A, lane 7). Phosphorylation at S847 increased fourfold to fivefold $(n=3$, $p \leq 0.0001$ ), reaching a plateau at 45 and $60 \mathrm{~min}$ (Fig. $5 A, B$ ); it was also sensitive to MK801 inhibition (Fig. $5 A$, lane 7).

\section{Phosphorylation of $\mathrm{nNOS}$ at $\mathrm{S} 1412$ regulates the surface expression of GLUT3}

Previously, we determined that phosphorylation of nNOS at S1412 by Akt was required for NMDAR-induced production of NO in neurons (Rameau et al., 2007). To determine whether phosphorylation of nNOS was coupled to GLUT3 trafficking, we examined the effects of an Akt inhibitor I (Akt-I), a phosphatidyl inositol ether analog, on NMDAR-induced expression of surface GLUT3. After stimulation, surface GLUT3 increased approximately fourfold relative to vehicle controls (Fig. 5D, lane 3, and $5 E)$. Akt-I blocked the increase in surface GLUT3 $(n=3, p \leq$ 0.001 ) (Fig. $5 D$, lane 2, and $5 E$ ), demonstrating that phosphorylation of nNOS by Akt is required for the coupling of NMDAR activity to the increase in surface GLUT3.

To confirm the role of the stimulatory, S1412 phosphorylation of nNOS in GLUT3 trafficking, we expressed the nNOS phosphomimetic mutant nNOS S1412D in cortical neurons. We isolated surface proteins by the biotin-streptavidin pull-down assay and determined the expression of surface GLUT3 by Western blotting. The expression of nNOS-S1412D increased surface GLUT3 by more than twofold ( $n=4 ; p \leq 0.003$ ) relative to GFP infection controls (Fig. $6 A, B$ ). In contrast, the expression of 
A
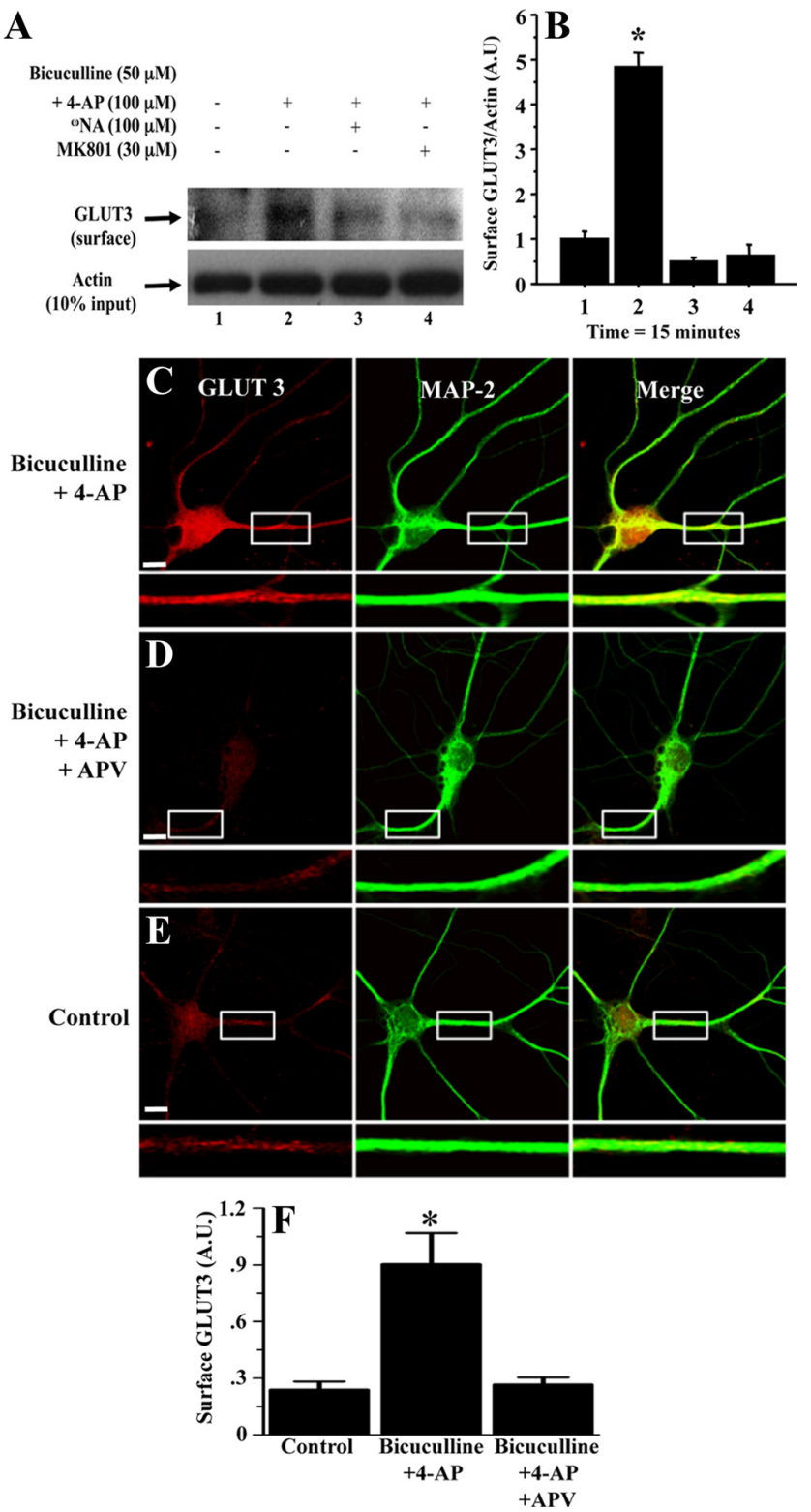

Figure 3. NMDAR regulates the surface expression of GLUT3 via production of NO. $A,{ }^{\omega} N A$ blocks NMDAR-mediated increased surface GLUT3. Western blotting for actin showed that equivalent levels of protein were loaded in each lane. $\boldsymbol{B}$, Quantification of $\boldsymbol{A}$, which showed that the ${ }^{\omega} \mathrm{NA}$ and MK801 significantly blocked NMDAR-induced surface expression of GLUT3 $(n=3$; $\left.{ }^{*} p=0.0025\right)$. Inhibition of NMDAR blocks the surface expression of GLUT3. C, Cultured hippocampal neurons immunostained for surface GLUT3 (red label; rhodamine) and MAP-2 (green label; fluorescein). D, Treatment with bicuculline plus 4-AP plus APV. $\boldsymbol{E}$, № drug, as control. $\boldsymbol{F}$, Quantification of surface GLUT3 in neuronal dendrites showed that activation of synaptic NMDAR increased surface GLUT3 $\left(n=50,{ }^{*} p<0.001\right)$. Scale bars, $20 \mu \mathrm{m}$.

wild-type nNOS showed no increase in surface GLUT3 relative to the GFP control. The effects of the phosphomimetic S1412D mutant on GLUT3 surface expression were not due to differential expression of the nNOS mutant since Western blot analyses had equivalent expression (Fig. 6A).

We also confirmed surface GLUT3 induction by quantitative confocal microscopy after expression of wild-type and S1412D mutant nNOS in hippocampal neurons. Expression of the S1412D mutant in hippocampal neurons increased surface GLUT3 levels twofold (Fig. 6C,F) $(n=45 ; p \leq 0.0001)$ relative to cells expressing wild-type nNOS or GFP as a control (Fig. 6D-F). These results support a model in which NMDAR transmission
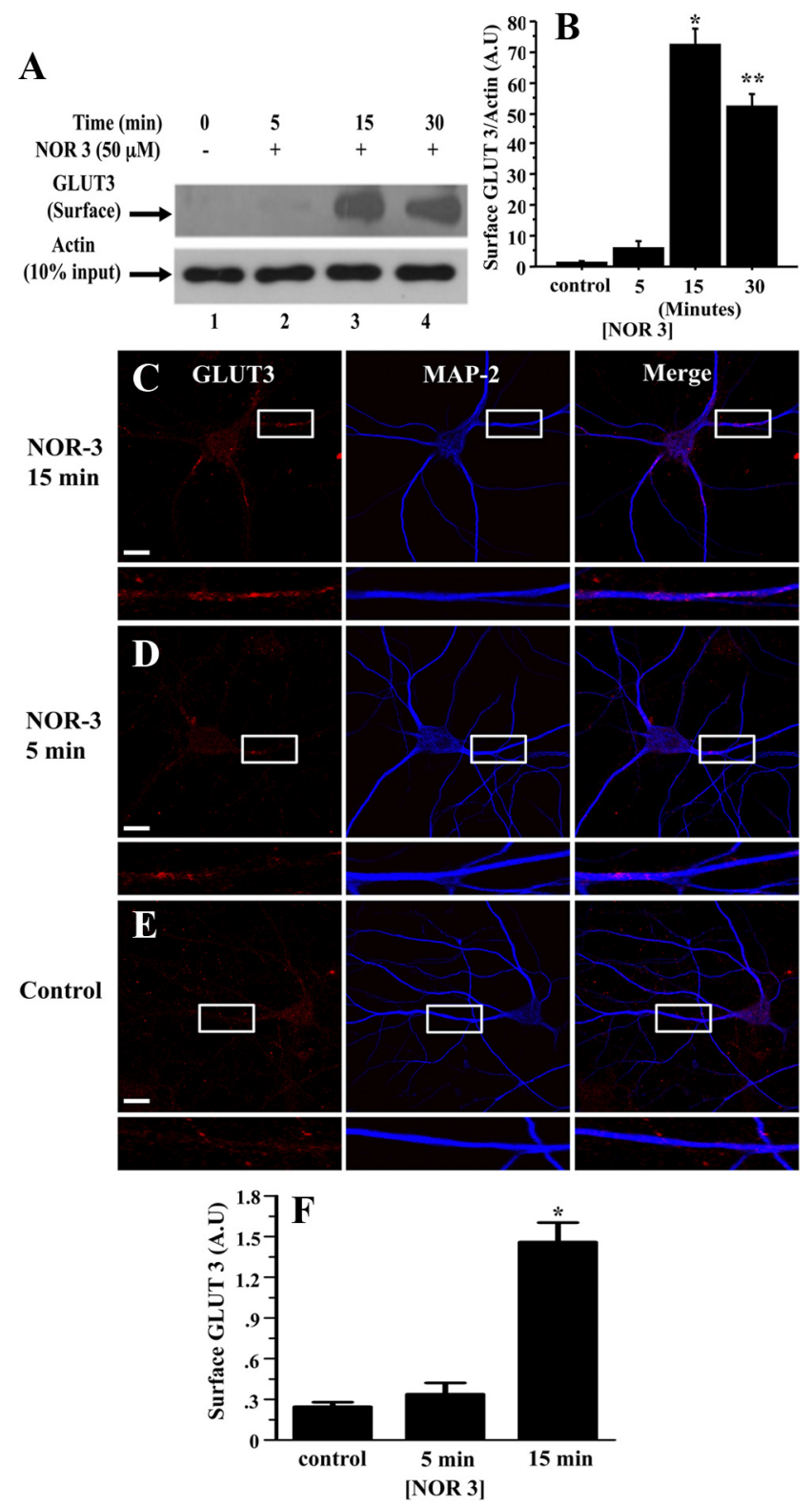

Figure 4. Increased release of N0 elevates the expression of surface GLUT3. A, Time course of increase in surface GLUT3. $B$, Quantification of $A$ expressed as the ratio of surface GLUT3 to actin $\left(n=3 ;{ }^{*} p<0.001\right)$. Immunostaining of hippocampal cultured neurons for surface GLUT3 (red label; rhodamine), and for MAP-2 (blue label; cyan 5) and merged images. C, D, Treatment with $50 \mu \mathrm{M}$ NOR 3 for $15 \mathrm{~min}(\boldsymbol{C})$ and with $50 \mu \mathrm{M}$ NOR 3 for $5 \mathrm{~min}(\boldsymbol{D})$. $\boldsymbol{E}$, No drug as control. $\boldsymbol{F}$, Quantification of surface GLUT3 $(n=50, p \leq 0.001)$. Scale bars, $20 \mu \mathrm{m}$.

activates nNOS via phosphorylation at S1412, and reinforces the notion that the increased production of NO regulates the expression of surface GLUT3.

\section{cGK activation mediates GLUT3 surface expression}

NO stimulates soluble GC (sGC), the enzyme that produces cGMP (Moncada and Higgs, 1991). In neurons, the stimulation of NMDAR increases cGMP (Garthwaite and Garthwaite, 1987), a principal mediator of NO signaling (Garthwaite and Boulton, 1995). To determine the role of cGMP production in GLUT3 trafficking, we examined the expression of surface GLUT3 induced by NMDAR in the presence of ODQ $(1 H-[1,2,4]$ oxadiazolo[4,3-a]quinoxalin-1-one), a potent inhibitor of sGC. Treatment with ODQ (50 $\mu \mathrm{M} ; 15 \mathrm{~min})$ blocked the NMDAR- 
A

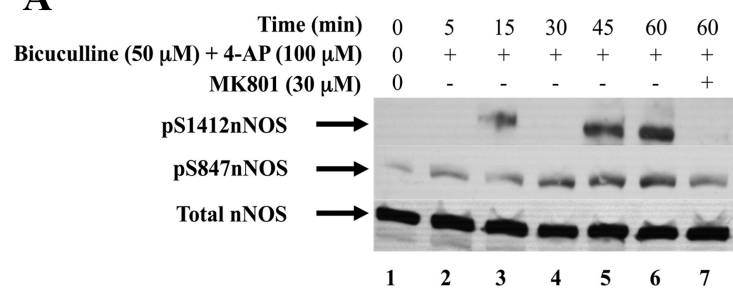

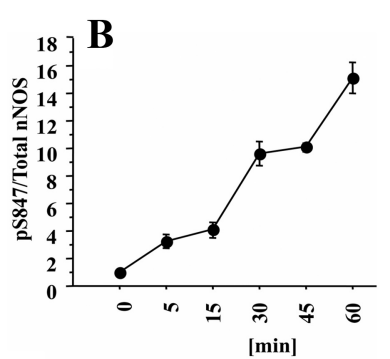

D

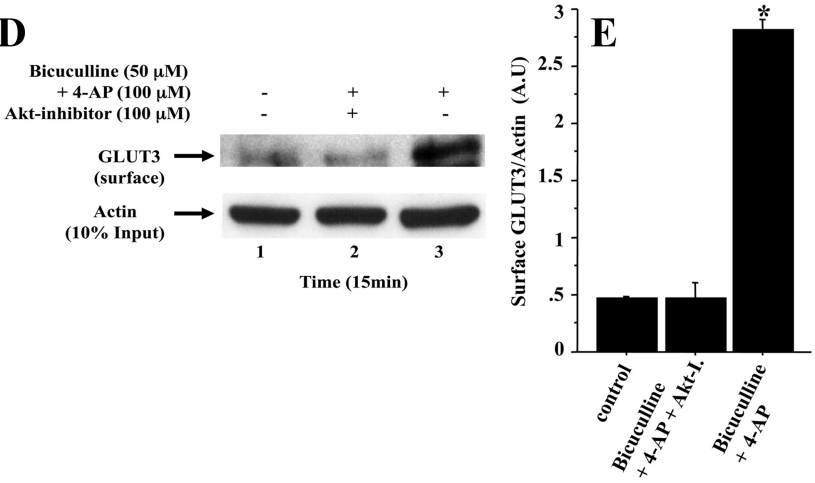

Figure 5. NMDAR stimulation regulates phosphorylation of nNOS at $S 847$ and $S 1412$ sites. $A$, Time course of increased phosphorylation of nNOS at S1412 and S847. B, C, Quantification of $A$, performed by taking the ratio of phospho-nNOS to total nNOS. D, Akt-mediated phosphorylation of $\mathrm{nNOS}$ increases surface expression of GLUT3. Surface GLUT3 induced by stimulation with bicuculline plus 4-AP for 15 min is blocked by Akt-I. Lane 1, no drug as control; lane 2, $50 \mu \mathrm{M}$ bicuculline plus $100 \mu \mathrm{m}$ 4-AP administered after preincubation with $100 \mu \mathrm{m}$ Akt-l; lane 3, 50 $\mu \mathrm{m}$ bicuculline plus $100 \mu \mathrm{m}$ 4-AP. $\boldsymbol{E}$, Quantification of $\boldsymbol{D}$ showed that Akt-I blocked NMDARinduced surface GLUT3. ${ }^{*} p<0.001$.

mediated increase in surface GLUT3, relative to the no-drug control $\left(n=3 ;{ }^{*} p \leq 0.005\right)$ (Fig. $7 A$, lanes 1,2 , and 4 , and $\left.7 B\right)$. ODQ inhibition of GLUT3 translocation was similar in magnitude to that seen with MK801 and ${ }^{\omega} \mathrm{NA}$ (Fig. 7A, lanes 3 and 5, and 7B). Increased cGMP regulates the activity of cGKs leading to an increase in surface GLUR1 (Serulle et al., 2007). Treatment with 8-Br-cGMP, a cell-permeable nonhydrolyzable form of cGMP, mimics the effect of cGMP on cGKs. To examine the role of cGKs in regulating the trafficking of GLUT3, neurons were treated with 8-Br-cGMP. Cultured hippocampal neurons were immunostained for GLUT3 and MAP-2. After treatment with $50 \mu \mathrm{M} 8$ 8-BrcGMP for $15 \mathrm{~min}$, surface GLUT3 increased eightfold $(n=50$, $p=0.001$ ) (Fig. 7C,F) relative to stimulation with $50 \mu \mathrm{M} 8$-BrcGMP plus Rp-8-pCPT-cGMPS, a potent cell-permeable cGK inhibitor or vehicle control (Fig. $7 D-F$ ). The results suggest that 8-Br-cGMP acts via cGK to increase the surface expression of GLUT3. However, the mechanism by which cGK activation regulates GLUT3 trafficking remains to be elucidated.

\section{NMDAR activation increases intracellular glucose}

We examined the coupling of synaptic activity to glucose uptake in cultured hippocampal neurons. We used the fluorescent tracer 2-NBDG, a substrate analog of glucose, which provides a readout
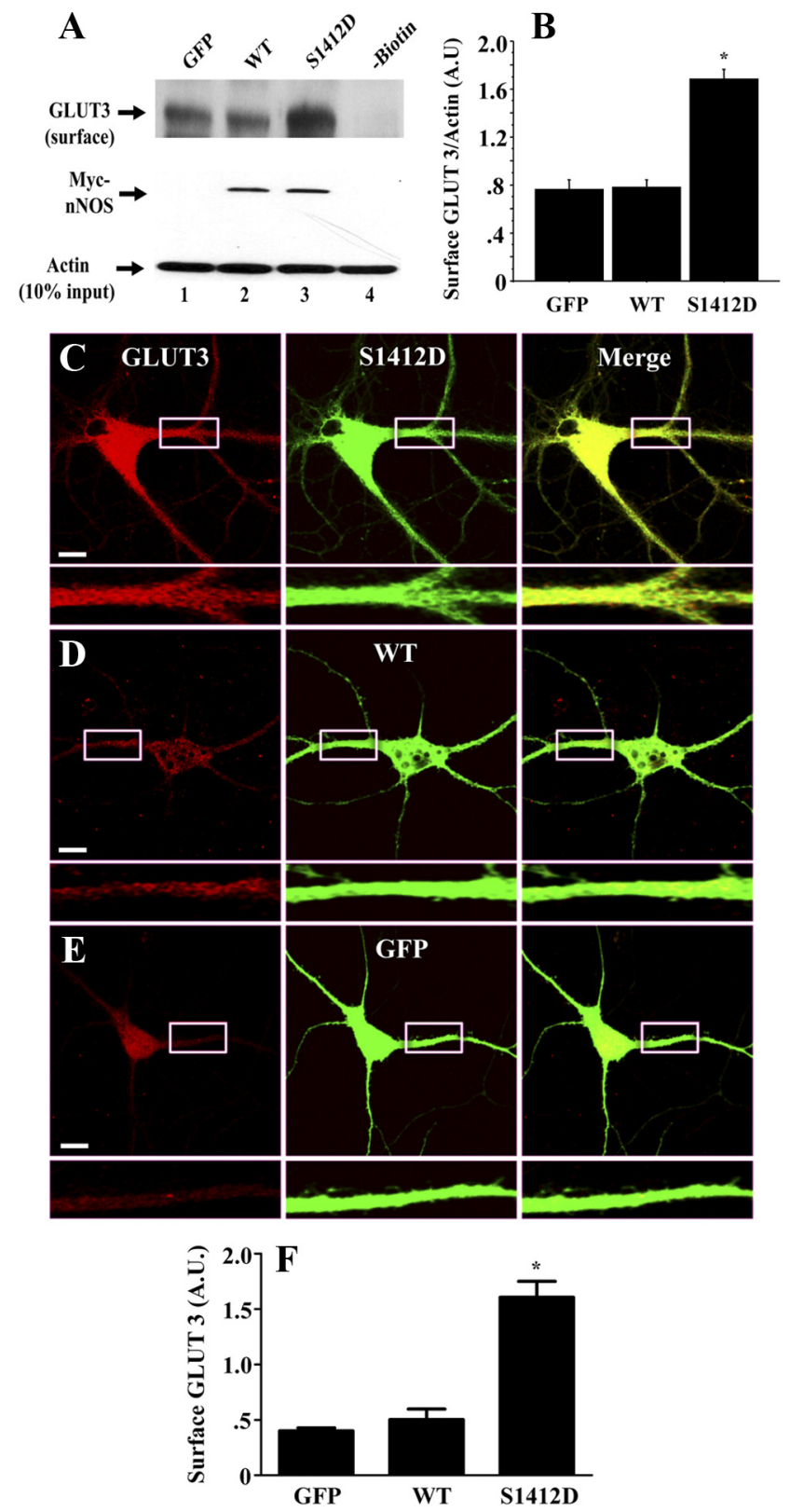

Figure 6. The expression of nNOS S1412D phosphomimetic mutant increases surface GLUT3. A, Surface GLUT3: lane 1, GFP, infection control; lane 2, wild-type nNOS; lane 3, S1412D; lane 4, no biotin, negative control for the biotin-streptavidin assay. $\boldsymbol{B}$, Quantification of $\boldsymbol{A}$ showed that exogenous expression of the S1412D mutant significantly increased surface GLUT3 relative to wild-type nNOS and GFP as control. ${ }^{*} p<0.003$. C $-\boldsymbol{E}$, Representative confocal images of hippocampal neurons showing surface GLUT3 levels (red label; rhodamine) and myc-epitopetagged nNOS (green label; fluorescein), phosphomimetic nNOS-S1412D (C), wild-type nNOS $(\boldsymbol{D})$, and GFP $(\boldsymbol{E})$ as control. $\boldsymbol{F}$, Quantification of surface GLUT3 showed that expression of nNOSS1412D displayed higher levels of surface GLUT3, fourfold relative to neurons expressing wildtype nNOS or GFP $\left(n=45,{ }^{*} p<0.0001\right)$. Scale bars, $20 \mu \mathrm{m}$.

of glucose uptake in neuronal cells (Porras et al., 2004). Stimulation of neurons with bicuculline plus 4-AP increased 2-NBDG uptake twofold (Fig. $8 D, J)(n=30, p \leq 0.0001)$ relative to vehicle control (Fig. $8 \mathrm{~A}, \mathrm{~J}$ ). A remarkable increase in the uptake and accumulation of the tracer over that of controls was seen in spines in cells treated with bicuculline (Fig. $8 A, D$ ). The appearance of 2-NBDG in spine structures after stimulation with bicuculline suggests that glucose transport occurs at synapses. Significantly, ${ }^{\omega} \mathrm{NA}$ blocked glucose uptake (Fig. 8G, J), demonstrating the de- 

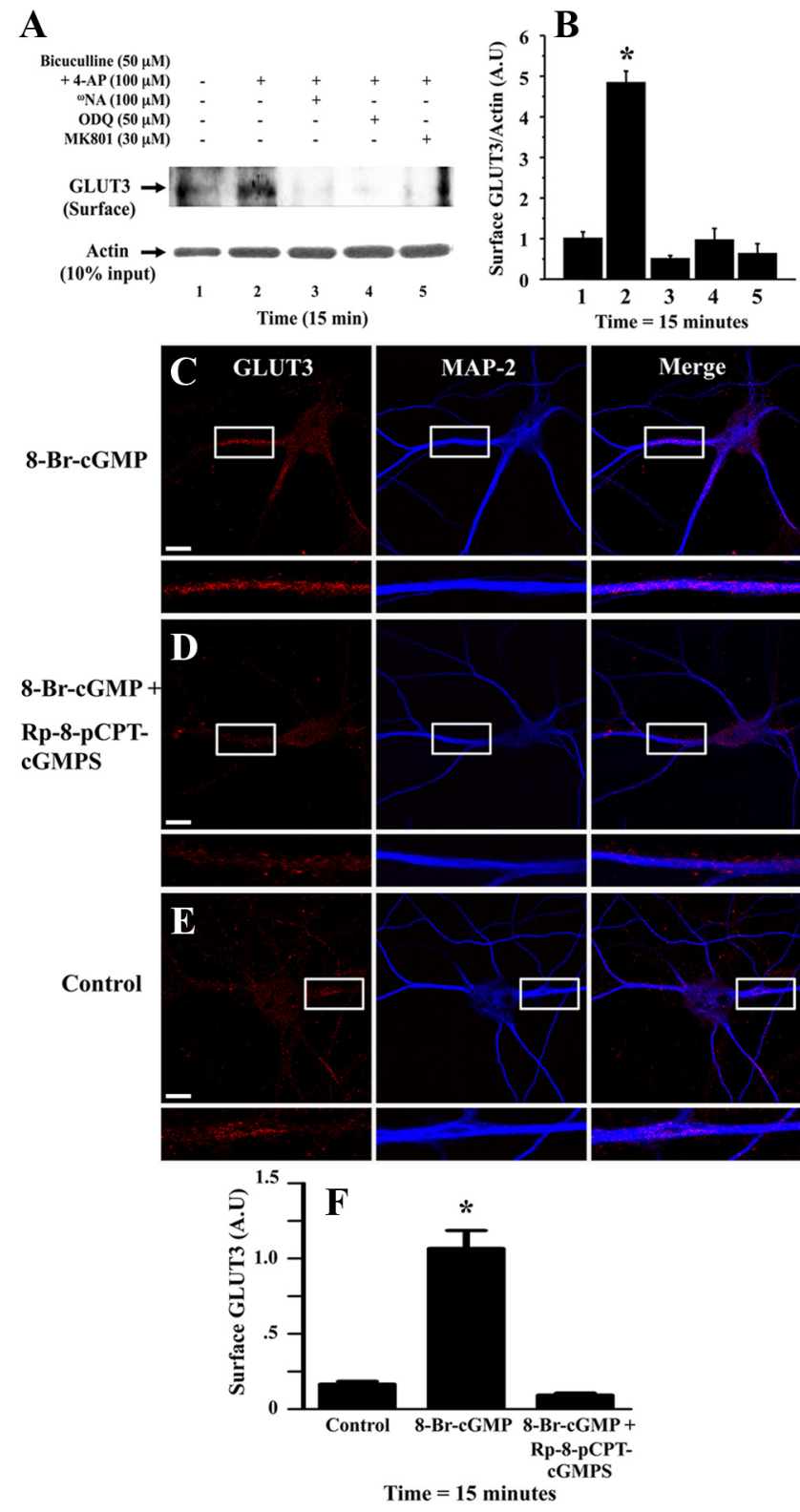

Figure 7. A cGK-dependent pathway increases the surface expression of GLUT3. A, Cortical cells were preincubated with ${ }^{\omega} \mathrm{NA}(100 \mu \mathrm{M}), \mathrm{DDQ}(100 \mu \mathrm{M})$, or MK-801 (30 $\left.\mu \mathrm{M}\right)$ for 15 min prior to the application of $50 \mu \mathrm{m}$ bicuculline plus $100 \mu \mathrm{m}$ 4-AP. Western blots for actin showed that equivalent levels of proteins were loaded in each lane. $\boldsymbol{B}$, Quantification of $\boldsymbol{A}$, which showed that $\mathrm{ODQ}{ }^{\omega} \mathrm{NA}$, and MK801 inhibited NMDAR-induced surface expression of GLUT3. Representative confocal images of neurons in which CGKs were stimulated with 8-Br-CGMP. ${ }^{*} p<0.005$. C-E, $50 \mu \mathrm{m}$ 8-Br-CGMP $(\boldsymbol{C}), 50 \mu \mathrm{m}$ 8-Br-CGMP plus $2 \mu \mathrm{m}$ Rp-8-pCPT-CGMPS (D), no drug $(\boldsymbol{E})$, as control. Surface GLUT3 (red label; rhodamine) and MAP-2 (blue label; cyan 5). $\boldsymbol{F}$, The bar graph shows that the fivefold increase in surface GLUT3 after treatment was blocked by Rp-8-pCPTcGMPS. ${ }^{*} p<0.005$.

pendence of glucose transport on a NO-mediated mechanism. Together, these results suggest that stimulation of synaptic NMDARs, activity of nNOS, and production of NO are tightly linked to the uptake of glucose.

\section{Discussion}

We examined activity-dependent regulation of surface GLUT3 in cultured neurons. We show that synaptic NMDAR activity increases surface GLUT3. This is in agreement with the long-held view that an increase in neuronal activity requires a readily avail-
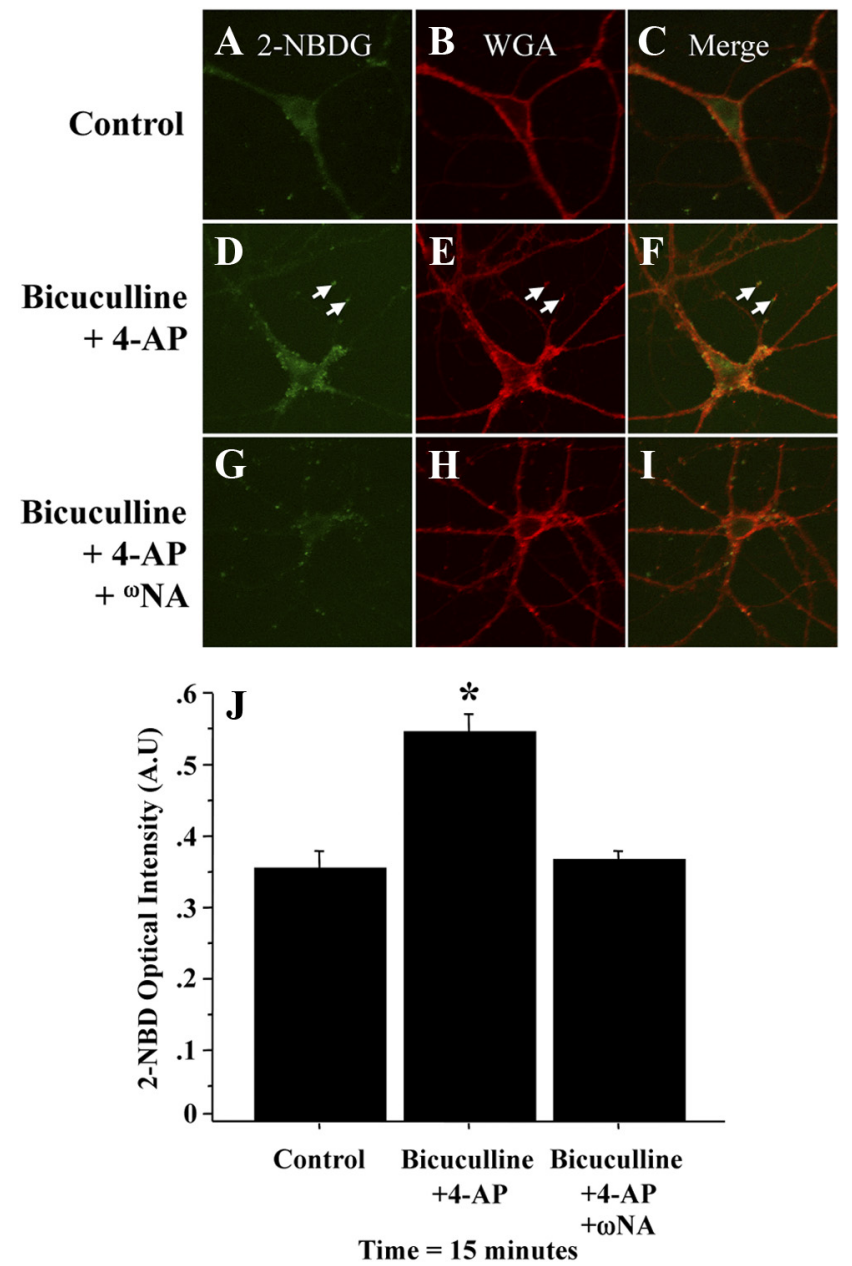

Figure 8. N0-mediated increase of surface GLUT3 elevates glucose transport. 2-NBDG levels were measured after increased neuronal activity and in the presence or absence of inhibitors of nNOS. A, D, G, Representative images obtained by confocal microscopy show 2-NBDG levels (green) for cells treated with no drug, as control, $50 \mu \mathrm{m}$ bicuculline plus $100 \mu \mathrm{m} 4$-AP and $50 \mu \mathrm{m}$ bicuculline plus $100 \mu \mathrm{m}$ 4-AP after preincubation with ${ }^{\omega} \mathrm{NA}$, respectively. Arrows show the accumulation of the tracer in dendritic spines. $\boldsymbol{B}, \boldsymbol{C}, \boldsymbol{E}, \boldsymbol{F}, \boldsymbol{H}, \boldsymbol{I}$, membrane labeling in red with WGA $(\boldsymbol{B}, \boldsymbol{E}, \boldsymbol{H})$ and merged images $(\boldsymbol{C}, \boldsymbol{F}, \boldsymbol{I})$, which showed that both the green and the red fluorescent staining belong to the same cell. J, Quantification of optical intensity of the fluorescent tracer 2-NBDG showed that glucose levels are increased after 15 min treatment with $50 \mu \mathrm{m}$ bicuculline plus $100 \mu \mathrm{m} 4$ 4-AP relative to control. ${ }^{\omega} \mathrm{NA}$ blocked the NMDAR-mediated increase in glucose. ${ }^{*} p<0.0001$

able energy substrate in the form of glucose (Keller et al., 1997). We also show that stimulation of NMDARs at synapses induces sequential stimulatory and inhibitory phosphorylation of nNOS that controls the production of NO. The inhibition of stimulatory phosphorylation of nNOS by Akt-I blocks the increase in surface GLUT3. This indicates a coupling of regulatory nNOS phosphorylation to glucose transport via increased production of NO that is required for the trafficking of GLUT3. The increase in surface GLUT3 after expression of phosphomimetic nNOS confirms the coupling of these events. The NMDAR-mediated increase in NO induces the cGMP/cGK-dependent signaling pathway that facilitates the translocation of GLUT3 to the plasma membrane. The increase in surface GLUT3 increased glucose transport, which is significantly reduced in the presence of a NOS inhibitor. Together, this study shows that synaptic activity regulates surface GLUT3, leading to increased glucose uptake in neuronal cells. 


\section{NMDAR induces production of NO} induces surface GLUT3

This study characterizes a novel relationship between the induction of neurotransmitter release at synapses and alterations in cellular GLUT3 surface expression. We show that stimulation of neurons with bicuculline plus 4-AP triggers a timedependent increase in surface expression of GLUT3. The GLUT3 surface expression increases significantly $15 \mathrm{~min}$ after transient stimulation of synaptic activity. Since the labeling of total GLUT3 shows a large dendritic pool relative to the surface GLUT3, this suggests the possibility that GLUT3 may be recruited from the dendritic pool. But, the mechanism that mediates the exocytosis of GLUT3 remains to be elucidated.

In HEK 293T, treatment with DETA-NO [(z)-1-[2-amino ethyl- $N$-(2-ammonioethyl) amino] diazen-lium-1,2-diolate], an NO donor, increases glucose transport and glycolysis via a mechanism that involves the activation of $5^{\prime}$-AMPactivated protein kinase and GLUT3 (Cidad et al., 2004). NMDARs transduce signals via the influx of $\mathrm{Ca}^{2+}$, which activates $\mathrm{Ca}^{2+}$ regulated targets, including nNOS. We show that the activation of NMDAR and subsequent increase in NO result in a rapid expression of surface GLUT3. To confirm the role of NO, we show that incubation with NOR-3 induces significant translocation of GLUT3 to the plasma membrane. These results suggest that a NO-dependent mechanism increases GLUT3 at the cell surface. However, the details of the mechanism by which NO regulates GLUT3 trafficking remain to be investigated.

\section{Coupling of phosphorylation of nNOS to synaptic NMDAR and regulation of surface GLUT3 by NO/cGK pathway}

Previously, we have shown that bath application of glutamate induces sequential phosphorylation of nNOS at S1412 and S847, respectively. Here, we show that synaptic NMDAR stimulation induces biphasic phosphorylation of nNOS at S1412 and a delayed increase in phosphorylation at S847, but phosphorylation at S1412 returns after an initial decline. The difference in the regulation of phosphorylation at S1412 in this paradigm reflects distinctive properties of synaptic NMDAR signaling cascades that induce differential regulation of kinase and phosphatase pathways for control of the phosphorylation of nNOS. Since, nNOS S1412 phosphorylation is required for $\mathrm{NO}$ production in neurons, we test the hypothesis that inhibition of phosphorylation at S1412 may block GLUT3 surface expression. We show that inhibition of Akt activity blocks the NOdependent increase in GLUT3 at cell surface. The expression of the phosphomimetic nNOS mutation S1412D provides an opportunity to test the sufficiency of $S 1412$ phosphorylation to increase the surface expression of GLUT3. The expression of nNOS mutant S1412D elevates GLUT3 at the plasma membrane, which suggests that stimulatory phosphorylation of nNOS regulates the expression of surface GLUT3. However, we cannot rule out the possibility that constitutive NO production imposes a metabolic burden on the cells, which triggers the increase in surface GLUT3. Given the multiple targets of NO function, it would be difficult to distinguish between several potential mechanisms including the reduction of $\mathrm{NADPH}$, arginine consumption, or protein phosphorylation. Although S1412D expression shows sufficiency and along with the Akt inhibition sug- gests that S1412 phosphorylation is involved, this does not establish that phosphorylation of S1412 is necessary for the surface expression of GLUT3.

We also show that increased GC activity facilitates the translocation of GLUT3 to the cell surface, since incubation with ODQ blocks NMDAR-induced surface expression of GLUT3. We also show that treatments with 8-Br-cGMP increase surface GLUT3. This implies that activation of a cGK regulates the expression of surface GLUT3. A precedent for cGKII mediating cellular trafficking was described for the regulation of AMPA receptor (Serulle et al., 2007). However, the trafficking step in which the activation of cGK exerts its effects on GLUT3 translocation to plasma membrane remains to be elucidated.

Finally, to monitor glucose uptake in neurons after induction of synaptic activity, we used the fluorescent tracer 2-NBDG. After stimulation with bicuculline, the tracer accumulates in dendritic buttons, which potentially correspond to sites of glutamate release at synapses. However, our results are at odds with a study in which glutamate treatment inhibited glucose transport in hippocampal neurons (Porras et al., 2004). The basis for these discrepancies may lie in part in the methodology of the experiments. In our study, cultured cortical neurons are treated with FdU. The astrocytes that grow in close proximity to neurons could confound the quantification of 2-NBDG in neurons. Significantly, we use a different stimulation protocol that targets synaptic NMDAR. The regulation of glucose uptake under these conditions might well be different from bath application of glutamate or NMDA where extrasynaptic NMDAR becomes stimulated. Further studies will be needed to clarify these issues.

In total, our results suggest a mechanism that regulates surface GLUT3 that may serve homeostatic strategies allowing neuronal cells to meet the changing energy demands brought about by increased synaptic activity. Specifically, synaptic stimulation of NMDARs through the regulation of phosphorylation of nNOS and a NO-dependent pathway induces the surface expression of GLUT3 and subsequent increase in glucose entry (Fig. 9). These results suggest that the regulation of glucose transport is fundamental to the neuronal adaptive response to increased synaptic activity, which is achieved by regulatory induction of the surface expression of GLUT3. 


\section{References}

Bredt DS, Snyder SH (1989) Nitric oxide mediates glutamate-linked enhancement of cGMP levels in the cerebellum. Proc Natl Acad Sci U S A 86:9030 9033.

Cidad P, Almeida A, Bolanos JP (2004) Inhibition of mitochondrial respiration by nitric oxide rapidly stimulates cytoprotective GLUT3-mediated glucose uptake through 5'-AMP-activated protein kinase. Biochem J 384: 629-636.

Dwyer DS (2001) Model of the 3-D structure of the GLUT3 glucose transporter and molecular dynamics simulation of glucose transport. Proteins 42:531-541.

Ganguly A, McKnight RA, Raychaudhuri S, Shin BC, Ma Z, Moley K, Devaskar SU (2007) Glucose transporter isoform-3 mutations cause early pregnancy loss and fetal growth restriction. Am J Physiol Endocrinol Metab 292:E1241-E1255.

Garthwaite J, Boulton CL (1995) Nitric oxide signaling in the central nervous system. Annu Rev Physiol 57:683-706.

Garthwaite J, Garthwaite G (1987) Cellular origins of cyclic GMP responses to excitatory amino acid receptor agonists in rat cerebellum in vitro. J Neurochem 48:29-39.

Garthwaite J, Garthwaite G, Palmer RM, Moncada S (1989) NMDA receptor activation induces nitric oxide synthesis from arginine in rat brain slices. Eur J Pharmacol 172:413-416.

Hardingham GE, Bading H (2002) Coupling of extrasynaptic NMDA receptors to a CREB shut-off pathway is developmentally regulated. Biochim Biophys Acta 1600:148-153.

Keller JN, Germeyer A, Begley JG, Mattson MP (1997) 17Beta-estradiol attenuates oxidative impairment of synaptic $\mathrm{Na}+/ \mathrm{K}+$-ATPase activity, glucose transport, and glutamate transport induced by amyloid betapeptide and iron. J Neurosci Res 50:522-530.

Khayat ZA, McCall AL, Klip A (1998) Unique mechanism of GLUT3 glucose transporter regulation by prolonged energy demand: increased protein half-life. Biochem J 333:713-718.

Lowe AG, Walmsley AR (1986) The kinetics of glucose transport in human red blood cells. Biochim Biophys Acta 857:146-154.
Moncada S, Higgs EA (1991) Endogenous nitric oxide: physiology, pathology and clinical relevance. Eur J Clin Invest 21:361-374.

Nagamatsu S, Sawa H, Kamada K, Nakamichi Y, Yoshimoto K, Hoshino T (1993) Neuron-specific glucose transporter (NSGT): CNS distribution of GLUT3 rat glucose transporter (RGT3) in rat central neurons. FEBS Lett 334:289-295.

Porras OH, Loaiza A, Barros LF (2004) Glutamate mediates acute glucose transport inhibition in hippocampal neurons. J Neurosci 24:9669-9673.

Rameau GA, Akaneya Y, Chiu L, Ziff EB (2000) Role of NMDA receptor functional domains in excitatory cell death. Neuropharmacology 39:2255-2266.

Rameau GA, Chiu LY, Ziff EB (2003) NMDA receptor regulation of nNOS phosphorylation and induction of neuron death. Neurobiol Aging 24:1123-1133.

Rameau GA, Tukey DS, Garcin-Hosfield ED, Titcombe RF, Misra C, Khatri L, Getzoff ED, Ziff EB (2007) Biphasic coupling of neuronal nitric oxide synthase phosphorylation to the NMDA receptor regulates AMPA receptor trafficking and neuronal cell death. J Neurosci 27:3445-3455.

Schmidt S, Richter M, Montag D, Sartorius T, Gawlik V, Hennige AM, Scherneck S, Himmelbauer H, Lutz SZ, Augustin R, Kluge R, Ruth P, Joost HG, Schürmann A (2008) Neuronal functions, feeding behavior, and energy balance in Slc2a3+/- mice. Am J Physiol Endocrinol Metab 295:E1084-E1094.

Serulle Y, Zhang S, Ninan I, Puzzo D, McCarthy M, Khatri L, Arancio O, Ziff EB (2007) A GluR1-cGKII interaction regulates AMPA receptor trafficking. Neuron 56:670-688.

Simpson IA, Davies P (1994) Reduced glucose transporter concentrations in brains of patients with Alzheimer's disease. Ann Neurol 36:800-801.

Simpson IA, Carruthers A, Vannucci SJ (2007) Supply and demand in cerebral energy metabolism: the role of nutrient transporters. J Cereb Blood Flow Metab 27:1766-1791

Zhao Y, Fung C, Shin D, Shin BC, Thamotharan S, Sankar R, Ehninger D, Silva A, Devaskar SU (2010) Neuronal glucose transporter isoform 3 deficient mice demonstrate features of autism spectrum disorders. Mol Psychiatry 15:286-299. 